\title{
The effect of lifestyle and risk factor modification on occlusive peripheral arterial disease outcomes: standard healthcare vs structured programme-for a randomised controlled trial protocol
}

\author{
M. Elfghi i* (D, F. Jordan², D. Dunne ${ }^{3}$, I. Gibson ${ }^{1,3}$, J. Jones ${ }^{3,4}$, G. Flaherty', S. Sultan ${ }^{5}$ and W. Tawfick ${ }^{1,5}$
}

\begin{abstract}
Background: Peripheral arterial disease (PAD) affects more than 200 million of the global population. PAD represents a marker for premature cardiovascular events. Patients with PAD, even in the absence of a history of myocardial infarction or ischemic stroke, have approximately the same relative risk of death from cardiovascular causes as patients with a history of coronary or cerebrovascular disease. Despite the high prevalence of PAD and the strong association with cardiovascular morbidity and mortality, patients with PAD are less likely to receive appropriate treatment for their atherosclerotic risk factors than those who are being treated for coronary artery disease.

Atherosclerotic risk factor identification and modification play an important role in reducing the number of adverse outcomes among patients with atherosclerosis. Risk reduction therapy decreases the risk of cardiovascular mortality and morbidity in patients with PAD. In this study, we aim to evaluate the effectiveness of a lifestyle and risk factor modification intervention programme in achieving treatment goals for PAD risk factors.
\end{abstract}

Methods: This is a randomised, parallel group, active-control trial to compare the effectiveness of the risk factor modification intervention programme to standard healthcare in a tertiary vascular care centre, in the reduction of modified risk factors in PAD patients. The primary outcome of this study is to evaluate the effectiveness of a lifestyle and risk factor modification intervention programme in achieving treatment goals for PAD risk factors at 3 and 12 months. The secondary outcomes are to compare the impact of the programme on clinical outcomes in PAD patients at 12 months. Secondary outcomes include amputation-free survival, clinical improvement, haemodynamic improvement, need for revascularisation procedures, outcomes of revascularisation procedures, changes in quality of life and the incidence of adverse events.

\footnotetext{
* Correspondence: marahelfghi@gmail.com

${ }^{1}$ School of Medicine, National University of Ireland, University Road, Galway, Ireland

Full list of author information is available at the end of the article
}

(c) The Author(s). 2021 Open Access This article is licensed under a Creative Commons Attribution 4.0 International License, which permits use, sharing, adaptation, distribution and reproduction in any medium or format, as long as you give appropriate credit to the original author(s) and the source, provide a link to the Creative Commons licence, and indicate if changes were made. The images or other third party material in this article are included in the article's Creative Commons licence, unless indicated otherwise in a credit line to the material. If material is not included in the article's Creative Commons licence and your intended use is not permitted by statutory regulation or exceeds the permitted use, you will need to obtain permission directly from the copyright holder. To view a copy of this licence, visit http://creativecommons.org/licenses/by/4.0/ The Creative Commons Public Domain Dedication waiver (http://creativecommons.org/publicdomain/zero/1.0/) applies to the data made available in this article, unless otherwise stated in a credit line to the data. 
(Continued from previous page)

Discussion: This study will provide clear evidence on the effectiveness of a lifestyle and risk factor modification intervention programme in achieving treatment goals for PAD risk factors, through a high-quality, well-powered clinical trial.

Trial registration: This trial was registered (11/07/2017) on the European Clinical Trials Database (EudraCT number 2017-002964-41) and ClinicalTrials.gov (NCT03935776) which was registered on 02 May 2019.

Keywords: Peripheral arterial disease, PAD, Atherosclerosis, Risk factors, Lifestyle and risk factor modification intervention programme, Randomised controlled trial

\section{Background}

Peripheral arterial disease (PAD) affects more than 200 million of the global population [1]. PAD represents a marker for premature cardiovascular events [2]. Patients with PAD, even in the absence of a history of myocardial infarction or ischemic stroke, have approximately the same relative risk of death from cardiovascular causes as patients with a history of coronary or cerebrovascular disease [2]. Despite the high prevalence of PAD and the strong association with cardiovascular morbidity and mortality, patients with PAD are less likely to receive appropriate treatment for their atherosclerotic risk factors than those who are being treated for coronary artery disease $[3,4]$.

As PAD represents a peripheral manifestation of atherosclerosis, most traditional and novel cardiovascular risk factors are strongly associated with this condition [5-7]. Smoking, diabetes, hyperlipidaemia, hypertension, unhealthy diet and physical inactivity were identified as significant modifiable risk factors that should be targeted for secondary prevention [8-12].

Atherosclerotic risk factor identification and modification play an important role in reducing the number of adverse outcomes among patients with atherosclerosis [13]. Risk reduction therapy decreases the risk of cardiovascular mortality and morbidity in patients with PAD [13]. Because of the efficacy of these techniques, several expert committees have recommended their use in patients with PAD [8-12]. Despite clear guidelines, several studies have shown that patients with PAD are routinely undertreated for these risk factors [4], which may contribute to high rates of morbidity and mortality.

Previous studies concluded that modifiable risk factor programmes help cardiac patients achieve their risk factor modification targets, with subsequent reduction in cardiovascular events [14-16]. To our knowledge, currently, there is still no evidence to support that the implementation of a structured modifiable risk factor reduction program will lead to improved outcomes in PAD patients. However, due to the similar atherosclerotic burden in cardiac and PAD patients, we hypothesise that these risk factor modification programmes would similarly improve risk factor target achievement in PAD patients and subsequently improve their clinical outcomes and reduce their amputation rates when compared to standard healthcare.

The possibility of lifestyle and risk factor modification intervention should have a positive impact on patients' clinical well-being and on their quality of life.

\section{Study objectives \\ Primary objective}

The primary objective of this study is to evaluate the effectiveness of a lifestyle and risk factor modification intervention programme in achieving treatment goals for PAD risk factors compared to the standard healthcare management.

\section{Secondary objectives}

Secondary objectives are to compare the impact of the programme on clinical outcomes in PAD patients, specifically:

- Amputation-free survival

- Clinical improvement [17]

- Haemodynamic improvement [17]

- Need for revascularisation procedures

- Changes in quality of life [18]

- The incidence of adverse events

\section{Methods}

\section{Study design}

This is a randomised, parallel group, active-control trial to compare the effectiveness of the risk factor modification intervention programme to standard healthcare in a tertiary vascular care centre, in the reduction of the prevalence of modifiable risk factors in PAD patients.

This trial will randomise patients with PAD in an equal ratio to one of two treatment arms. One arm will be randomised to undergo a risk factor modification intervention programme at a community-based centre. The other arm will be provided with standard healthcare advice, in the outpatient PAD clinic in a tertiary referral vascular centre. Patients randomised into the risk factor 
modification intervention programme will have the intervention administered for 12 weeks. Patients will then be followed for 12 months to determine further PAD outcomes. The participant flowchart through the study is shown in Fig. 1. SPIRIT figure for this trial is given in Fig. 2.

\section{Study setting}

Potential participants will be identified from the outpatient PAD clinic at the University Hospital Galway,
Ireland (UHG). Patients will be screened and randomised at the outpatient PAD clinic in UHG.

The risk factor modification intervention programme will be administered in a nurse-led community-based centre (Croí Heart and Stroke Centre, Galway, Ireland), in the presence of a physiotherapist and dietitian. The control arm will be managed in the outpatient PAD clinic, UHG. The coordinating centre will be the Department of Vascular and Endovascular Surgery, UHG, and the School

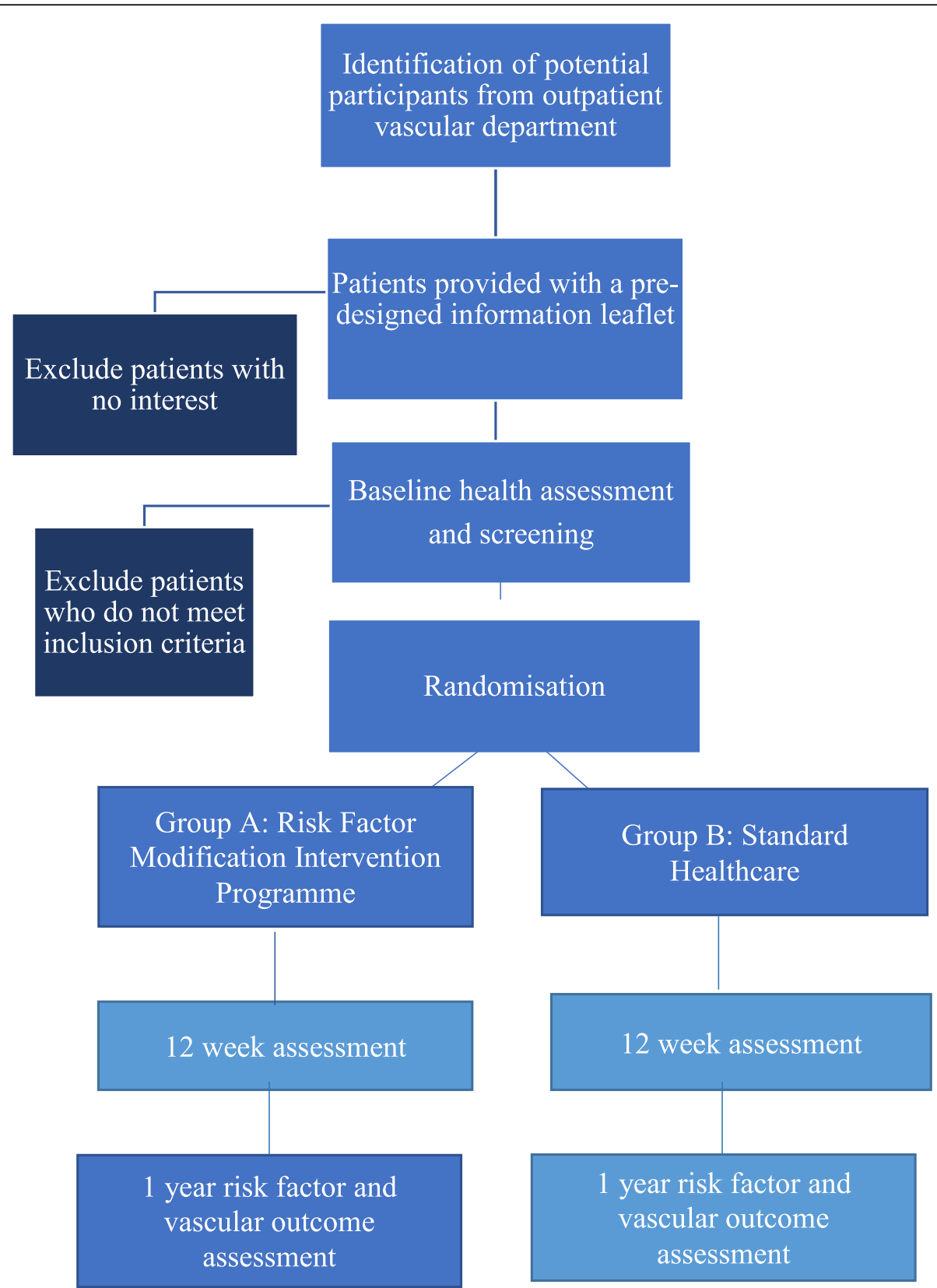

Fig. 1 Participant flowchart through the study 


\begin{tabular}{|c|c|c|c|c|c|c|}
\hline \multirow[b]{2}{*}{ TIME POINT } & \multirow{2}{*}{$\begin{array}{c}\text { Enrolment } \\
- \text { t1 }\end{array}$} & \multirow{2}{*}{$\begin{array}{c}\text { Allocation } \\
0\end{array}$} & \multicolumn{4}{|c|}{ Study Period } \\
\hline & & & $\begin{array}{l}\text { Baseline } \\
\text { Week 0 }\end{array}$ & $\begin{array}{l}\text { Intervention } \\
\text { (12 weeks) }\end{array}$ & $\begin{array}{l}12 \text { weeks } \\
\text { follow up }\end{array}$ & $\begin{array}{l}12 \text { months } \\
\text { follow up }\end{array}$ \\
\hline \multirow{3}{*}{$\begin{array}{c}\text { Eligibility screen } \\
\text { Informed consent } \\
\text { Randomisation }\end{array}$} & $\mathbf{x}$ & & & & & \\
\hline & $\mathbf{x}$ & & & & & \\
\hline & & $\mathbf{x}$ & & & & \\
\hline \multirow{3}{*}{\multicolumn{7}{|c|}{$\begin{array}{c}\text { INTERVENTIONS: } \\
\text { Risk Factor Modification } \\
\text { Intervention Programme } \\
\text { Standard Healthcare }\end{array}$}} \\
\hline & & & & & & \\
\hline & & & & & & \\
\hline \multicolumn{7}{|l|}{ ASSESSMENTS: } \\
\hline $\begin{array}{l}\text { PAD assessment: ABI, } \\
\text { digital pressure, } \\
\text { Rutherford category, and } \\
\text { WIfI }\end{array}$ & & & $\mathbf{x}$ & & $\mathbf{x}$ & $\mathbf{x}$ \\
\hline $\begin{array}{l}\text { Blood samples including: } \\
\text { Fasting blood lipids, } \\
\text { Fasting glucose and } \\
\text { HbA1c }\end{array}$ & & & $\mathbf{X}$ & & $\mathbf{X}$ & $\mathbf{X}$ \\
\hline $\begin{array}{l}\text { Blood pressure } \\
\text { documentation }\end{array}$ & & & $\mathbf{x}$ & & $\mathbf{x}$ & $\mathbf{x}$ \\
\hline $\begin{array}{l}\text { Anthropometric } \\
\text { measurements including: } \\
\text { BMI and Waist } \\
\text { Circumference }\end{array}$ & & & $\mathbf{x}$ & & $\mathbf{x}$ & $\mathbf{x}$ \\
\hline $\begin{array}{l}\text { Physical capacity } \\
\text { including: Shuttle test, } \\
\text { Claudication distance } \\
\text { and Absolute walking } \\
\text { distance }\end{array}$ & & & $\mathbf{x}$ & & $\mathbf{x}$ & $\mathbf{x}$ \\
\hline HADS & & & $\mathbf{x}$ & & $\mathbf{x}$ & $\mathbf{x}$ \\
\hline $\begin{array}{l}\text { Dartmouth Quality of Life } \\
\text { score }\end{array}$ & & & $\mathbf{x}$ & & $\mathbf{x}$ & $\mathbf{x}$ \\
\hline $\begin{array}{l}\text { Fagerstrom Test for } \\
\text { Nicotine } \quad \text { Dependence }\end{array}$ & & & $\mathbf{x}$ & & $\mathbf{x}$ & $\mathbf{x}$ \\
\hline $\begin{array}{l}\text { Godin and Shepard } \\
\text { Leisure Exercise } \\
\text { Questionnaire }\end{array}$ & & & $\mathbf{x}$ & & $\mathbf{x}$ & $\mathbf{x}$ \\
\hline $\begin{array}{l}\text { Nutritional assessment } \\
\text { through the } \\
\text { Mediterranean Diet } \\
\text { Questionnaire }\end{array}$ & & & $\mathbf{x}$ & & $\mathbf{x}$ & $\mathbf{x}$ \\
\hline major amputation & & & & & & $\mathbf{x}$ \\
\hline $\begin{array}{l}\text { revascularisation or a } \\
\text { reintervention }\end{array}$ & & & & & & $\mathbf{x}$ \\
\hline intervention or stenosis & & & & & & $\mathbf{X}$ \\
\hline MACE & & & & & & $\mathbf{X}$ \\
\hline MALE & & & & & & $\mathbf{x}$ \\
\hline $\begin{array}{l}\text { Health related quality of } \\
\text { life }\end{array}$ & & & & & & $\mathbf{X}$ \\
\hline $\begin{array}{l}\text { Cost-effectiveness of the } \\
\text { programme }\end{array}$ & & & & & & $\mathbf{X}$ \\
\hline
\end{tabular}

Fig. 2 SPIRIT figure showing an overview of the assessment schedule at baseline and follow-up in study. ABl: ankle-brachial Index, WIfl: wound ischaemia and foot infection classification, HbA1c: glycosylated haemoglobin, BMI: body mass index, HADS: Hospital Anxiety and Depression Scale, MACE: major adverse cardiovascular event and MALE: major adverse limb event

of Medicine at the National University of Ireland Galway (NUI Galway). Patients are directly supervised during the intervention. The 12 -week and 1-year assessments will take place at the outpatient PAD clinic in UHG.

\section{Eligibility criteria Inclusion criteria}

- Aged 18 years or more

- Provide written informed consent 
- PAD: diagnosed by at least one of the following:

- Ankle-brachial index of less than 0.90 in at least one lower extremity [9]

- Toe brachial index of less than 0.60 [9]

- Evidence of arterial occlusive disease in one lower extremity detected by duplex ultrasonography, computed tomographic angiography, or magnetic resonance angiography [9]

- Symptomatic PAD (Rutherford category 2 and above [19])

- Patients should have at least one of the following risk factors:

- Blood pressure $>140 / 80 \mathrm{mmHg}$

- Fasting blood sugar $(\mathrm{FBS})>53 \mathrm{mmol} / \mathrm{mol}$

- Glycosylated haemoglobin (HbA1c) > 7\%

- Total cholesterol $>5 \mathrm{mmol} / \mathrm{L}$

- Low-density lipoprotein (LDL) cholesterol > 2.6 $\mathrm{mmol} / \mathrm{L}$

- Triglycerides $>1.7 \mathrm{mmol} / \mathrm{L}$

- High-density lipoprotein $(\mathrm{HDL})<1.0 \mathrm{mmol} / \mathrm{L}$ in men and $<1.2 \mathrm{mmol} / \mathrm{L}$ in women

- Physical activity less 30 min for 5 days per week

- Body mass index (BMI) $>25 \mathrm{~kg} / \mathrm{m}^{2}$

- Waist circumference $>80 \mathrm{~cm}$ in women, and $>94$ $\mathrm{cm}$ in men

- Current smoker or exposure to tobacco in any form

- Unhealthy diet, Mediterranean diet score less than 10 points

\section{Exclusion criteria}

- Involvement in another clinical trial in the previous 6 months

- Legal incapacity

- Inadequate English language ability to understand the content of the intervention programme

- Significant cognitive impairment or mental illness

- Refusal to participate in a certain part of the intervention

- Patient suffering from a comorbidity that could affect either their physical participation in the intervention arm or influence outcomes (e.g. ischemic heart failure or severe chronic kidney disease with an estimated glomerular filtration rate of less than $30 \mathrm{~mL} / \mathrm{min}$ [20])

- Patient is immobile

- Contraindication to anticoagulation and antiplatelet medications or any of the risk factors treatment.

\section{Study screening}

Patients with Symptomatic PAD (Rutherford category 2 and above [19]) will be invited to join the study. Invited patients will be provided with a pre-designed information leaflet. This leaflet will be fully explained to the patient at the initial assessment. The study researchers will answer any questions about the study. Informed consent will be obtained from the patient on a formatted consent form. Patients will be given the freedom to give consent either on the same day or at a later date in accordance with a study within a trial (SWAT), entitled "Same-day Consent vs Delayed Consent in a Randomised Trial: A Study within a Trial" [21]. This SWAT aims to ensure the rigorousness of the consent process and will run in conjunction with this randomised controlled trial.

Researchers will screen the patient for inclusion and exclusion criteria and administer a series of the following:

1. Record PAD risk factors such as smoking, hyperlipidaemia, diabetes, hypertension, increased body weight and the patient's current medication.

2. Document the Rutherford category [19], claudication distance and absolute walking distance for each patient, to assess the severity of PAD.

3. Schedule appointments for baseline health assessments over the following month.

\section{Randomisation}

After meeting the inclusion criteria, screened patients will be randomised to one of two treatment arms. One arm will receive the 12-week intensive risk factor modification intervention programme. The control arm will be provided with standard care in the outpatient PAD clinic. This is an intention to treat designed study, where patients are analysed as randomised. Each screened patient will be given a unique screening number.

Screened patients will be randomised in a 1:1 ratio of study intervention: control according to a randomisation scheme. The randomisation scheme will be produced using the PROC PLAN $^{\circ}$ procedure of the $\mathrm{SAS}^{\circ}$ software package (version 9.2.2) using a simple randomisation strategy. The scheme will be concealed from all patients and study personnel until after database lock.

Patients will be allocated to intervention via an automated telephone system, which will not deliver the randomised allocation except after registering the subject screening number. Each screened patient who is recruited to the trial will be given a unique patient trial number.

The statistician will remain blinded to the treatment allocation until all the data have been analysed to minimise bias. Outcome assessors and data analysts will be blinded; however, in the event of an adverse event outcome, assessors will be unblinded. 


\section{Baseline, prior to intervention}

All randomised patients will undergo a full baseline assessment prior to their intervention:

1. PAD assessment;

a. Ankle-brachial index (ABI)

b. Digital pressure

c. Rutherford category [19]

d. Wound ischaemia and foot infection classification (WIfI) [22]

2. Blood samples including:
a. Fasting blood lipids
b. Fasting glucose
c. HbA1c

3. Blood pressure documentation

4. Anthropometric measurements including:

a. BMI

b. Waist circumference

5. Sub-maximal functional capacity exercise testing including:

a. Shuttle test [23]

b. Claudication distance [24]

c. Absolute walking distance [25]

6. Behavioural and psychological survey using the Hospital Anxiety and Depression Scale (HADS) [26].

7. Health-related quality of life assessment: Dartmouth Quality of Life score [18].

8. Smoking status assessment using the Fagerstrom Test for Nicotine Dependence [27].

9. Physical activity assessment: Godin and Shepard Leisure Exercise Questionnaire [28].

10. Nutritional assessment through the Mediterranean Diet Questionnaire [29].

\section{Intervention}

\section{Risk factor modification structured Programme}

The risk factor modification intervention programme is a 12-week intensive lifestyle programme. This is a nurseled, community-based, lifestyle and risk factor modification intervention modelled on the European Society of Cardiology demonstration project, a large clinical trial called EuroAction [30]. The programme includes:

- Phase 1: Initial individualised assessment by the multidisciplinary team (MDT) will include previously mentioned baseline assessment in addition to the following:

- Dietician will assess, current eating habits and food diary

- Exercise specialist will assess 7-day activity recall, barriers to exercise, 7-day pedometer and Functional Capacity Test
- Phase 2: The intervention including:

- Weekly exercise class and educational workshops.

- Serial blood pressure, body mass index, waist circumference, glucose and lipid measurements with goal setting.

- Weekly MDT meetings.

- Targeted and protocol-based pharmacotherapy to support lifestyle changes.

\section{Standard healthcare}

The control group will receive the standard healthcare advice provided to PAD patients in the outpatient PAD clinic. In this study, standard care will be conducted by the researchers which include:

- Advising patients to quit smoking, regular exercise and healthy eating, but neither structured intervention nor organised cessation plans will be addressed.

- Non-specific interventions, such as providing patients with educational material on general health problems.

\section{Twelve-week assessment}

On completion of the 12 weeks in both groups, patients are reassessed for risk factors, therapeutic management and lifestyle changes similar to baseline.

\section{One-year assessment}

Similar to the baseline and 12-week assessment, in addition to clinical outcomes assessment, the following are included:

1. If the patient underwent a major amputation and level of amputation

2. If required a revascularisation procedure.

3. Any residual stenosis of more than $30 \%$ [17]

4. If developed a major adverse cardiovascular event (MACE) or major adverse limb event (MALE)

5. Health-related quality of life

6. Cost-effectiveness of the programme

\section{Endpoints}

\section{Primary endpoint}

Achieving target improvement in lifestyle risk factors at 12 weeks and at 1 year. Target improvement will be considered if the patient achieves any one or more of the following:

1. Smoking cessation

2. BMI $20-25\left(\mathrm{~kg} / \mathrm{m}^{\wedge} 2\right)$. BMI is calculated by dividing body weight in kilogrammes by the square of height in meters 
3. HbA1c less than $7 \%$

4. Total cholesterol less than $5.0 \mathrm{mmol} / \mathrm{L}$

Patients will be reassessed for the primary endpoint at 1 year. If they fail the criteria by which they were deemed at 12 weeks to have achieved the primary endpoint, they will then be considered to not have achieved the 1-year primary endpoint.

We will report the primary endpoint for both time points.

\section{Secondary endpoints}

Secondary endpoints of PAD outcomes are based on the Society for Vascular Surgery (SVS) reporting standards [17]:

1. Amputation-free survival; defined as time spent free from any major above ankle amputation [17]

2. Hemodynamic improvement: defined as an increase in the ABI by at least 0.10 [17]

3. Clinical improvement: which is defined as an upward shift by at least one Rutherford category. However, PAD patients suffering from actual tissue loss (Rutherford category 5 [19]) should move up two Rutherford categories to be considered improved [19].

4. Re-intervention or stenosis rate; any re-intervention or stenosis among patients who already underwent vascular surgery [17]

5. Freedom from major adverse cardiovascular events (MACE). MACE is defined as any major cardiovascular event such as myocardial infarction, cerebrovascular accident or death [17].

6. Major adverse limb events (MALE). MALE is defined as any major amputation or revascularization procedure [17].

7. Revascularisation-free survival; defined as time free from any revascularisation procedure regardless of if it were an endovascular intervention or an open surgery [17].

8. Change from baseline of the following risk factors:

a- BMI (measured in $\mathrm{kg} / \mathrm{m}^{2}$ )

b- HbA1c (measured as a percentage)

c- Total cholesterol (measured in $\mathrm{mmol} / \mathrm{L}$ )

9. Health-related quality of life [18]; assessed using the Dartmouth Cooperative Information Project (COOP) charts at enrolment and after 1 year. The COOP charts measure six core aspects of functional status: physical fitness, feelings, daily activities, social activities, change in health, pain, and overall health. The instrument consists of six charts, referring to the abovementioned aspects of functioning. Each chart consists of a simple title, a question referring to the status of the patient and an ordinal 5-point response scale illustrated with a simple drawing. Each item is rated on this 5-point ordinal scale ranging from 1 (no limitation at all) to 5 (severely limited); for "change in health" score 1 means "much better" and score 5 "much worse". The designers do not advocate summing the responses to gain a single index figure of health status

\section{Safety endpoints}

1. Incidence and severity of adverse events

2. Incidence of side effects due to medication commenced during the trial, for the modulation of any PAD risk factor. This includes any drug interaction of these medications with any previously assumed medication the patient was using regularly prior to commencing the trial.

\section{Sample size calculation}

For sample size calculation, the EUROACTION study [30] was used to estimate the coefficient of variation for sample proportions.

Data from the EUROACTION [30] study suggest that 12-week intervention response rates for the primary endpoint of 54.8\% (intervention programme) and 35.6\% (usual care). Eighty percent statistical power and an alpha level of $5 \%$ were chosen. A two-sample comparison of proportions sample size calculation was implemented.

With these parameters, the G*Power [31] software yields a trial with a maximum sample size of 208 patients completing the intervention (104 per intervention group).

\section{Statistical analysis}

All data will be analysed according to the intention to treat principle. The primary outcome, the achievement of treatment goals for PAD risk factors between both groups at 12 weeks and 12 months will be compared using chi-square or Fisher's exact where appropriate. Secondary outcomes including time to event will be assessed using Kaplan-Meier survival curves and log rank test. An exact 95\% confidence interval will be applied for the difference between intervention groups in terms of PAD risk factor reduction.

\section{Discussion}

PAD is a very common disease that affects the quality of life of a large segment of the global population [1]. While PAD has been associated with increased risk of cardiovascular events, yet most recommendations for risk factor modulation in this population has been based mainly on studies on patients with cardiovascular disease [14-16, 32]. 
These recommendations for lifestyle modification techniques in PAD patients have included smoking cessation based on level 1B evidence and healthy diet and physical activity based on level $1 \mathrm{C}$ evidence [12]. However, none of these recommendations was based on randomised clinical trials. In fact, the recommendation for smoking cessation in PAD patients was based on observational studies that noted that smokers had a sevenfold increased risk of developing PAD [33, 34] and a two-fold higher risk of amputation [35]. There are no randomised controlled trials that have quantified the direct effect of smoking cessation, directly targeting this specific PAD population.

It is worth noting that most PAD patients have multifactorial risk factors. This adds to the limitations of any study designed to target risk factor modulation. Studies designed with a single risk factor treatment approach have the potential to fail, due to the confounding effects of other risk factors. Alternatively, in a study like our proposed trial, where a full multi-risk factor improvement approach is adopted, it is difficult to assess, which particular intervention yielded the best outcome.

In a multifactorial risk factor modification programme, some of the risk factors, like blood pressure, blood tests, anthropometrics and exercise testing assessments, are easily assessed with objective measurement tools. However, certain aspects can only be assessed using subjective methods like the HADS [26], Dartmouth Quality of Life score [18], Fagerstrom Test for Nicotine Dependence [27] and Mediterranean Diet Questionnaire [29]. This is a potential limitation of this study, due to the inherent bias of patients responding to such subjective questionnaires.

It has been shown that patients with PAD are routinely undertreated for most risk factors [4], which may contribute to high rates of morbidity and mortality. However, in the absence of level 1 evidence to support its implementation in this particular cohort of patients, it could be difficult to convince health authorities of the benefits of spending on such programmes. Cost of providing a multifactorial risk factor modification programme to all PAD patients could be prohibitive. However, if proven to be effective and included in the guidelines of management of PAD patients, this added cost should be weighed against the potential benefits and long-term savings of reducing cardiovascular events. We aim with this study to provide clear evidence for the effectiveness of a lifestyle and risk factor modification intervention programme in achieving treatment goals for PAD risk factors, through a high-quality, well-powered clinical trial.

Any important protocol modifications will be communicated first to the REC. Following ethical approval, an amended patient information leaflet will be circulated to trial participants. The trial registries and journal will be notified of the amended protocol.

\section{Trial registration}

This trial was registered (11/07/2017) on the European Clinical Trials Database (EudraCT number 2017002964-41) and ClinicalTrials.gov (NCT03935776) which was registered on 02 May 2019. Additional file 1; Section 1, shows Trial Registration Data Set.

\section{Trial status}

The study is ongoing at the time of submitting this manuscript (November 2019). This trial was using protocol version 2.0 (14 March 2018) at the time of this submission. Recruitment started in the University College Hospital, Galway, Ireland on 1 June 2018 and is expected to be completed on 1 June 2021. The trial management committee manages and disseminates the protocol amendments.

\section{Supplementary Information}

The online version contains supplementary material available at https://doi. org/10.1186/s13063-021-05087-x

Additional file 1:. Section 1. Trial Registration Data Set. Section 2. Consent Form for the RCT. Section 3. Consent Form Checklist for the RCT

\section{Abbreviations}

ABI: Ankle-brachial index; AE: Adverse event; BMI: Body mass index; BRPM: Blinded report planning meeting; CRF: Case report form; CTA: Clinical Trial Authorisation; FAS: Full Analysis (data) Set; HADS: Hospital Anxiety and Depression Scale; HbA1c: Glycosylated haemoglobin; HDL: High-density lipoprotein; ICH: International Conference on Harmonisation; LDL: Lowdensity lipoprotein; MACE: Major adverse cardiovascular event; MALE: Major adverse limb event; MDT: Multidisciplinary team; NUI: National University of Ireland, Galway; PAD: Peripheral arterial disease; PP: Per protocol; SAE: Serious adverse event; SAP: Statistical analysis plan; SVS: Society for Vascular Surgery; UCHG: University College Hospital, Galway, Ireland; WIfl: Wound ischaemia and foot infection classification

\section{Acknowledgements}

We would like to acknowledge the support provided by the National Institute for Prevention and Cardiovascular Health and the Croí Heart and Stroke Centre, Galway, Ireland.

\section{Authors' contributions}

WT conceived and led the study design. ME, DD, IG, JJ, GF, WT and SS contributed to the design of the study and protocol. ME and WT are responsible for data management. ME drafted the manuscript. Steering committee includes WT, SS and GF. Endpoint adjudication committee includes WT, GF and FJ. The data monitoring committee will be GF, JJ and $I G$, they will review the collected data for completeness and accuracy, and they are independent from the sponsor. All authors contributed to manuscript revision and read and approved the final manuscript.

\section{Funding}

As sponsor of one of the co-investigators medical doctorate scholarship, Ministry of Higher Education and Scientific Research, Libyan Embassy will provide funding for the trial. The funding organisation will have no involvement in the design and conduct of the study; collection, management, analysis, and interpretation of the data; preparation, review or approval of the manuscript; or the decision to submit the manuscript for publication. 


\section{Availability of data and materials}

The final datasets underlying publications, resulting from this trial, will be shared as an anonymous copy upon reasonable and approved request. A request may be made through email to the Principal Investigator and can only be made upon meeting the terms and conditions for the ethics approval of this trial.

\section{Ethics approval and consent to participate}

Ethical approval has been obtained from the Merlin Park Hospital, Clinical Research Ethics Committee (approval number: C.A. 1912).

The procedures detailed in this protocol are designed to ensure that investigators abide by the principles of the Good Clinical Practice guidelines of the International Conference on Harmonisation $(\mathrm{ICH})$. The trial will adhere to the guidelines of the World Medical Association Declaration of Helsinki in its revised edition (Fortaleza 2013) [36], as well as the demands of national drug and data protection laws and other applicable regulatory requirements. The investigator is responsible for ensuring that no patient is subject to any trial related examination or activity before that patient has given informed consent. Written consent must be given by the patient after the receipt of detailed information. The verbal explanation will cover all the elements specified in the written information provided for the patient. The investigator will inform the patient of the aims, methods, anticipated benefits and potential hazards of the study including any discomfort it may entail. The patient must be given every opportunity to clarify any points he/ she does not understand and if necessary, as for more information. Patients will be required to sign and date the informed consent form. Participants will also be asked for permission for the research team to share relevant data with people from the Universities taking part in the research or from regulatory authorities, where relevant. This trial does not involve collecting biological specimens for storage. After completion, informed consent forms will be kept and archived by the investigator in the investigator's trial master file. We are conducting a study within the trial Study within a Trial "Same-day Consent vs Delayed Consent in a Randomised Trial: A Study within a Trial", to ensure of the rigorousness of the consent process [21].

It should be emphasised that the patient is at liberty to withdraw their consent to participate at any time, without penalty or loss of benefits to which the patient is otherwise entitled. Patients who refuse to give, or who withdraw written informed consent will not be included or continued in the trial; however, they will be assured of continued appropriate medical care.

\section{Consent for publication}

Not applicable.

\section{Competing interests}

The authors declare that they have no competing interests.

\section{Author details}

'School of Medicine, National University of Ireland, University Road, Galway, Ireland. ${ }^{2}$ School of Nursing and Midwifery, National University of Ireland, University Road, Galway, Ireland. ${ }^{3}$ National Institute for Prevention and Cardiovascular Health, Croi Heart and Stroke Centre, Mayola Lane, Newcastle, Galway, Ireland. ${ }^{4}$ Brunel University, Kingston Ln, Uxbridge, London UB8 3PH, UK. ${ }^{5}$ Department of Vascular and Endovascular Surgery, University College Hospital, Galway (UCHG), Newcastle Road, Galway, Ireland.

\section{Received: 1 December 2019 Accepted: 1 February 2021}

\section{Published online: 13 February 2021}

\section{References}

1. Fowkes FGR, Rudan D, Rudan I, Aboyans V, Denenberg JO, McDermott MM, et al. Comparison of global estimates of prevalence and risk factors for peripheral artery disease in 2000 and 2010: a systematic review and analysis. Lancet. 2013;382(9901):1329-40.

2. Newman AB, Shemanski L, Manolio TA, Cushman M, Mittelmark M, Polak JF, et al. Ankle-arm index as a predictor of cardiovascular disease and mortality in the cardiovascular health study. Arterioscler Thromb Vasc Biol. 1999;19(3): 538-45.

3. Hirsch AT, Criqui MH, Treat-Jacobson D, Regensteiner JG, Creager MA, Olin $J W$, et al. Peripheral arterial disease detection, awareness, and treatment in primary care. Jama. 2001;286(11):1317-24.
4. Becker GJ, McClenny TE, Kovacs ME, Raabe RD, Katzen BT. The importance of increasing public and physician awareness of peripheral arterial disease. J Vasc Interv Radiol. 2002;13(1):7-11.

5. Criqui MH, Fronek A, Klauber MR, Barrett-Connor E, Gabriel S. The sensitivity, specificity, and predictive value of traditional clinical evaluation of peripheral arterial disease: results from noninvasive testing in a defined population. Circulation. 1985;71(3):516-22.

6. Ix JH, Allison MA, Denenberg JO, Cushman M, Criqui MH. Novel cardiovascular risk factors do not completely explain the higher prevalence of peripheral arterial disease among African Americans: The San Diego Population Study. J Am Coll Cardiol. 2008;51(24):2347-54.

7. Newman AB, Siscovick DS, Manolio TA, Polak J, Fried LP, Borhani NO, et al. Ankle-arm index as a marker of atherosclerosis in the cardiovascular health study. Cardiovascular Heart Study (CHS) Collaborative Research Group. Circulation. 1993;88(3):837-45.

8. Hirsch AT, Haskal ZJ, Hertzer NR, Bakal CW, Creager MA, Halperin JL, et al. ACC/AHA 2005 Practice Guidelines for the management of patients with peripheral arterial disease (lower extremity, renal, mesenteric, and abdominal aortic). Circulation. 2006;113(11)::463-654.

9. Norgren L, Hiatt WR, Dormandy JA, Nehler MR, Harris KA, Fowkes FGR, et al. Inter-society consensus for the management of peripheral arterial disease (TASC II). Eur J Vasc Endovasc Surg. 2007;33(1):S1-S75.

10. Rooke TW, Hirsch AT, Misra S, Sidawy AN, Beckman JA, Findeiss LK, et al. 2011 ACCF/AHA focused update of the guideline for the management of patients with peripheral artery disease (updating the 2005 guideline). Vasc Med. 2011;16(6):452.

11. Conte MS, Pomposelli FB, Clair DG, Geraghty PJ, McKinsey JF, Mills JL, et al. Society for Vascular Surgery practice guidelines for atherosclerotic occlusive disease of the lower extremities: management of asymptomatic disease and claudication. J Vasc Surg. 2015;61(3):2S-41S.

12. Piepoli MF, Hoes AW, Agewall S, Albus C, Brotons C, Catapano AL, et al. 2016 European Guidelines on cardiovascular disease prevention in clinical practice The Sixth Joint Task Force of the European Society of Cardiology and Other Societies on Cardiovascular Disease Prevention in Clinical Practice (constituted by representatives of 10 societies and by invited experts) Developed with the special contribution of the European Association for Cardiovascular Prevention \& Rehabilitation (EACPR). Eur Heart J. 2016;37(29): 2315-81.

13. Lu J, Creager $\mathrm{M}$. The relationship of cigarette smoking to peripheral arterial disease. Rev Cardiovasc Med. 2003:5(4):189-93.

14. Kotseva K, Connolly SB, Jennings C, Mead A, Jones J, Holden A, et al. EUROACTION: An ESC project in preventive cardiology programme in coronary and high risk patients and their partners; 2007. p. 285.

15. Wood D, Kotseva K, Jennings C, Mead A, Jones J, Holden A, et al. EUROACTION: a European Society of Cardiology demonstration project in preventive cardiology - a cluster randomised controlled trial of a multidisciplinary preventive cardiology programme for coronary patients, asymptomatic high risk individuals and their families. Summary of design, methodology and outcomes. Eur Heart J Suppl. 2004;6(J):J3-J15.

16. Kotseva K, Wood D, De Backer G, De Bacquer D, Pyörälä K, Keil U, et al. Cardiovascular prevention guidelines in daily practice: a comparison of EUROASPIRE I, II, and III surveys in eight European countries. Lancet. 2009; 373(9667):929-40.

17. Stoner MC, Calligaro KD, Chaer RA, Dietzek AM, Farber A, Guzman RJ, et al. Reporting standards of the Society for Vascular Surgery for endovascular treatment of chronic lower extremity peripheral artery disease. J Vasc Surg. 2016;64(1):e1-e21.

18. McHorney CA, Ware JE Jr, Rogers W, Raczek AE, Lu JR. The validity and relative precision of MOS short-and long-form health status scales and Dartmouth COOP charts: results from the Medical Outcomes Study. Med Care. 1992:MS253-MS65.

19. Rutherford RB, Baker JD, Ernst C, Johnston KW, Porter JM, Ahn S, et al. Recommended standards for reports dealing with lower extremity ischemia: revised version. J Vasc Surg. 1997;26(3):517-38.

20. Lewis R. Understanding chronic kidney disease: a guide for the nonspecialist. Cumbria: M\&K Update Ltd; 2012.

21. Elfghi M, Jordan F, Tawflck W. SWAT 84: Same-day consent vs delayed consent in a randomised trial. The Northern Ireland Network for Trials Methodology Research. 2018. online, available at: https://www.qub.ac.uk/ sites/TheNorthernlrelandNetworkforTrialsMethodologyResearch/FileStore/ Filetoupload,996661,en.pdf. [Accessed on 12/3/2018]. 
22. Mills JL, Conte MS, Armstrong DG, Pomposelli FB, Schanzer A, Sidawy AN, et al. The society for vascular surgery lower extremity threatened limb classification system: risk stratification based on wound, ischemia, and foot infection (WIfI). J Vasc Surg. 2014;59(1):220-34. e2

23. Liu NY-S, Plowman SA, Looney MA. The reliability and validity of the 20meter shuttle test in American students 12 to 15 years old. Res Q Exerc Sport. 1992;63(4):360-5.

24. Kruidenier LM, Nicolaï SP, Willigendael EM, de Bie RA, Prins MH, Teijink JA. Functional claudication distance: a reliable and valid measurement to assess functional limitation in patients with intermittent claudication. BMC Cardiovasc Disord. 2009;9(1):9.

25. Fabry R, Dubost J-J, Pochon P, Duchene-Marullaz P. Comparison of standard one-minute treadmill exercise and strandness test (absolute walking distance) in relation to site of lesion, walking distance, and diastolic blood flow velocity (Doppler curves). Angiology. 1990:41(10):869-76.

26. Zigmond AS, Snaith RP. The hospital anxiety and depression scale. Acta Psychiatr Scand. 1983;67(6):361-70.

27. Heatherton TF, Kozlowski LT, Frecker RC, Fagerstrom KO. The Fagerström test for nicotine dependence: a revision of the Fagerstrom Tolerance Questionnaire. Addiction. 1991;86(9):1119-27.

28. Godin G, Shephard R. Godin leisure-time exercise questionnaire. Med Sci Sports Exerc. 1997:29(6):36-8.

29. Martinez-Gonzalez M, Fernandez-Jarne E, Serrano-Martinez M, Wright M, Gomez-Gracia E. Development of a short dietary intake questionnaire for the quantitative estimation of adherence to a cardioprotective Mediterranean diet. Eur J Clin Nutr. 2004:58(11):1550-2.

30. Wood DA, Kotseva K, Connolly S, Jennings C, Mead A, Jones J, et al. Nursecoordinated multidisciplinary, family-based cardiovascular disease prevention programme (EUROACTION) for patients with coronary heart disease and asymptomatic individuals at high risk of cardiovascular disease: a paired, cluster-randomised controlled trial. Lancet. 2008;371(9629):19992012.

31. Faul F, Erdfelder E, Lang A-G, Buchner A. G* Power 3: a flexible statistical power analysis program for the social, behavioral, and biomedical sciences. Behav Res Methods. 2007;39(2):175-91.

32. Gibson I, Flaherty G, Cormican S, Jones J, Kerins C, Walsh AM, Costello C, Windle J, Connolly S, Crowley J. Translating guidelines to practice: findings from a multidisciplinary preventive cardiology programme in the west of Ireland. Eur J Prev Cardiol. 2014;21(3):366-76.

33. Shammas NW. Epidemiology, classification, and modifiable risk factors of peripheral arterial disease. Vasc Health Risk Manag. 2007;3(2):229.

34. Price J, Mowbray Pl, Lee AJ, Rumley A, Lowe GD, Fowkes FG. Relationship between smoking and cardiovascular risk factors in the development of peripheral arterial disease and coronary artery disease; Edinburgh Artery Study: Edinburgh Artery Study. Eur Heart J. 1999;20(5):344-53.

35. Bendermacher BL, Willigendael EM, Teijink JA, Prins MH. Medical management of peripheral arterial disease. J Thromb Haemost. 2005;3(8): 1628-37.

36. General Assembly of the World Medical Association. World Medical Association Declaration of Helsinki: ethical principles for medical research involving human subjects. J Am Coll Dentists. 2014;81(3):14.

\section{Publisher's Note}

Springer Nature remains neutral with regard to jurisdictional claims in published maps and institutional affiliations.

\section{Ready to submit your research? Choose BMC and benefit from}

- fast, convenient online submission

- thorough peer review by experienced researchers in your field

- rapid publication on acceptance

- support for research data, including large and complex data types

- gold Open Access which fosters wider collaboration and increased citations

- maximum visibility for your research: over $100 \mathrm{M}$ website views per year

At $\mathrm{BMC}$, research is always in progress.

Learn more biomedcentral.com/submissions 